(C) 1998 by the authors. Reproduction of this article by any means, is permitted for non-commercial purposes.

Adv. Theor. Math. Phys. 2 (1998) 533-558

\title{
The complete set of ground states of the ferromagnetic XXZ chains
}

\author{
Tohru Koma ${ }^{a, 1}$, Bruno Nachtergaele $e^{b, 2}$ \\ ${ }^{a}$ Department of Physics \\ Gakushuin University \\ Mejiro, Toshima-ku \\ Tokyo 171, JAPAN \\ ${ }^{b}$ Department of Mathematics \\ University of California, Davis \\ Davis, CA 95611-8633, U. S. A.
}

\begin{abstract}
We show that the well-known translation invariant ground states and the recently discovered kink and antikink ground states are the complete set of pure infinite-volume ground states (in the sense of local stability) of the spin- $S$ ferromagnetic XXZ chains with Hamiltonian $H=-\sum_{x}\left[S_{x}^{(1)} S_{x+1}^{(1)}+S_{x}^{(2)} S_{x+1}^{(2)}+\Delta S_{x}^{(3)} S_{x+1}^{(3)}\right]$, for all $\Delta>1$, and all $S \in \frac{1}{2} \mathbb{N}$. For the isotropic model $(\Delta=1)$ we show that all ground states are translation invariant.

For the proof of these statements we propose a strategy for demonstrating completeness of the list of the pure infinite-volume ground states of a quantum many-body system, of which the present results for the XXX and XXZ chains can be seen as an example. The result for $\Delta>1$ can also be proved by an easy extension to general $S$ of the method used in [T. Matsui, Lett. Math. Phys. 37 (1996) 397] for the spin-1/2 ferromagnetic XXZ chain with $\Delta>1$. However, our proof is
\end{abstract}

e-print archive: http://xxx.lanl.gov/abs/cond-math/9709208

${ }^{1}$ E-mail: koma@riron.gakushuin.ac.jp

${ }^{2}$ E-mail: bxn@math.ucdavis.edu 
different and does not rely on the existence of a spectral gap. In particular, it also works to prove absence of non-translationally invariant ground states for the isotropic chains $(\Delta=1)$, which have a gapless excitation spectrum.

Our results show that, while any small amount of the anisotropy is enough to stabilize the domain walls against the quantum fluctuations, no boundary condition exists that would stabilize a domain wall in the isotropic model $(\Delta=1)$.

\section{Introduction}

In this paper we consider the ground states of the spin- $S$ ferromagnetic XXZ chains with formal Hamiltonian

$$
H=-\sum_{x}\left[S_{x}^{(1)} S_{x+1}^{(1)}+S_{x}^{(2)} S_{x+1}^{(2)}+\Delta S_{x}^{(3)} S_{x+1}^{(3)}\right]
$$

for all $\Delta \geq 1$, and all $S \in \frac{1}{2} \mathbb{N}$. Our main objective is to show that the known ground states of these models are the complete set of ground states in the sense of stability under local perturbations. In the isotropic case $(\Delta=1)$ all ground states are translation invariant. For the anisotropic ferromagnetic XXZ chains $(\Delta>1)$, non-translation invariant ground states (kinks and antikinks) were recently discovered, independently by Alcaraz, Salinas, and Wreszinski [1] and Gottstein and Werner [2]. See Section 3 for a detailed description of these states.

Before we describe in detail our results, we explain the somewhat surprising insights that the detailed study of the XXZ ferromagnets has brought along. We believe that from this and other simple models one can obtain interesting information about the nature and the effects of quantum fluctuations, with relevance for a variety of phenomena in magnetism and interface physics.

In the absence of an external field and at low temperatures real ferromagnets usually exhibit domain wall structures. But it is commonly believed that pinning effects due to impurities and defects are crucial to stabilize domain walls against quantum fluctuations [3-7]. In other words, in models of quantum ferromagnetism that do not incorporate impurities or defects, one should not expect to find stable domain walls because they are destroyed by quantum fluctuations. While pinning may still be important to keep the domain walls from wandering around, the results for the XXZ Heisenberg model mentioned above, show that an arbitrary small amount of anisotropy is sometimes enough to stabilize interfaces against the quantum fluctuations.

A natural question, which is the subject of this paper, is whether or not one can obtain other ground states, e.g., that have interfaces with a different geometry or internal structure. Is it possible to construct more 
infinite-volume ground states, e.g., by imposing radically different boundary conditions and taking thermodynamic limits? The standard mathematical formulation of this question is in terms of the local stability condition: what is the complete set of infinite-volume ground states $\omega$ satisfying

$$
\lim _{\Lambda \rightarrow \mathbb{Z}} \omega\left(A^{*}\left[H_{\Lambda}, A\right]\right) \geq 0, \quad \text { for all } A \in \mathcal{A}_{\mathrm{loc}} ?
$$

The notations are explained and more details are given in Section 2. See [8] for an informative discussion of the problem of obtaining all states by imposing different boundary conditions.

The first result in this direction is due to Matsui [9]. For the spin- $1 / 2$ XXZ chain with $\Delta>1$, he proved that any pure infinite-volume ground state is either the all spins up, the all spins down, a kink, or an antikink state, i.e., the set of states given in [1] and [2] is the complete set. Matsui's proof, however, relies on rather special properties of the model. In particular, his method cannot be used to treat the isotropic (XXX) ferromagnetic chain which has a gapless excitation spectrum. The most important contribution in this paper is the proof that all the ground states of the XXX ferromagnetic chain are translation invariant. Our strategy also gives a new proof of completeness in the case of the XXZ chains, for all values of the spin. We also believe that our method can be adapted to other models. Absence of non-translation invariant ground states for the AKLT model [10] was recently proved in [11].

It is often said that the local stability definition of ground state (1.2), although natural both from the theoretical and experimental point of view, is of little practical use, because, until recently, it seemed impossible to find all its solution for a non-trivial quantum model. Even in cases where there is general agreement about what the full set of ground states should be, it was impossible to show that the known set of ground states is indeed the complete set (see the comments at the end of Section 6.2 in volume 2 of [12]). It remains true that extracting interesting information from the local stability inequalities alone, is usually quite difficult. But we hope to convince the reader that by combining it with other ideas (zero-energy states) and by using equivalent formulations, such as the one due to Bratteli, Kishimoto, and Robinson (see Theorem 2.3), interesting progress can be made.

Our result for the XXX chains should not be confused with another, very interesting, completeness result for the XXX ferromagnetic chain. In [13] Babbitt and Thomas proved that the Bethe Ansatz states lead to a complete resolution of the identity (Plancherel formula) in the GNS representation of any of its pure translation invariant ground states. These two completeness results nicely complement each other.

The present paper is organized as follows: In Section 2 we present our main results (Theorem 2.1 for the anisotropic and Theorem 2.2 for the 
isotropic chains). We also give a schematic description of the proof. In the proofs we use some properties of the finite-volume and infinite-volume zero-energy ground states of the XXZ chains. These are presented in Section 3. Section 4 is devoted to the proof of Theorem 2.1. The proof of Theorem 2.2 is in Section 5.

\section{Main results and structure of the proof}

In this section we limit ourselves to present a precise statement of the main results and an outline of the proof. More detailed definitions and notations, as well as additional results, are discussed in the next section.

A natural way of defining a quantum spin model is by specifying its Heisenberg dynamics, or, equivalently, the generator of this dynamics, $\delta$, given as a densely defined closed operator on the algebra of quasi-local observables of the spin chain. For a chain of spin- $S$ degrees of freedom, the algebra of quasi-local observables $\mathcal{A}$ is the $\mathrm{C}^{*}$-inductive limit (三 the completion for the operator norm) of the strictly local observables

$$
\mathcal{A}_{\mathrm{loc}}=\bigcup_{\Lambda \subset \mathbb{Z}} \mathcal{A}_{\Lambda}, \quad \mathcal{A}_{\Lambda}=\bigotimes_{x \in \Lambda} M_{\mathbb{C}}(2 S+1)
$$

where the union is over finite subsets of the integers, and $M_{\mathbb{C}}(2 S+1)$ denotes the complex $(2 S+1) \times(2 S+1)$ matrices.

The local Hamiltonians and the basis observables are usually expressed in terms of the spin matrices $S_{x}^{(1)}, S_{x}^{(2)}, S_{x}^{(3)}$, which are the generators of the $(2 S+1)$-dimensional irreducible unitary representation of $\mathrm{SU}(2)$. The subscript $x \in \mathbb{Z}$ refers to the site in the chain, i.e., the factor in the tensor product, with which these matrices are associated. The local Hamiltonians for the ferromagnetic XXZ chains are given by

$$
-\sum_{x=-L}^{L-1}\left[S_{x}^{(1)} S_{x+1}^{(1)}+S_{x}^{(2)} S_{x+1}^{(2)}+\Delta S_{x}^{(3)} S_{x+1}^{(3)}\right]=H_{L} \in \mathcal{A}_{[-L, L]} .
$$

Here $\Delta$ is the anisotropy parameter and we will always assume $\Delta \geq 1$. The generator of the dynamics is then defined by

$$
\delta(A)=\lim _{L \rightarrow \infty}\left[H_{L}, A\right]
$$

in the sense that

$$
A(t)=\exp (i t \delta) A, \quad \text { for all } A \in \mathcal{A} .
$$


By definition, a state $\omega$, i.e., a positive normalized linear functional, on $\mathcal{A}$ is a ground state of the model iff

$$
\omega\left(A^{*} \delta(A)\right)=\lim _{L \rightarrow \infty} \omega\left(A^{*}\left[H_{L}, A\right]\right) \geq 0, \quad \text { for all } A \in \mathcal{A} .
$$

This inequality expresses the property that any local perturbation of $\omega$ has a total energy at least equal to the energy of the unperturbed state. So, ground states minimize the energy locally.

The solutions of (2.5) can be described as follows:

1. If $\Delta=1$ (the isotropic case), all solutions are translation invariant. The support of the finite-volume restrictions of any ground state is contained in the subspace of maximal total spin. This means that these states are, in fact, permutation invariant. In the case $S=1 / 2$, they are all permutation invariant states. For $S>1 / 2$, there are permutation invariant states that are not ground states.

2. If $\Delta>1$, there are two translation invariant ground states, $\omega_{\uparrow}$ and $\omega_{\downarrow}$, which are characterized by the property

$$
\omega_{\uparrow}\left(S_{x}^{(3)}\right)=+S, \quad \omega_{\downarrow}\left(S_{x}^{(3)}\right)=-S, \text { for all } x \in \mathbb{Z} .
$$

In addition to these two, there are two infinite sets of pure nontranslation invariant ground states, called kinks and antikinks. The kink ground states approach $\omega_{\uparrow}$ as $x \rightarrow-\infty$ and $\omega_{\downarrow}$ as $x \rightarrow+\infty$. For antikink states the roles of plus and minus infinity are reversed.

These ground states were all known from the previous works [1,2]. For more detailed properties we refer to the next section. In this paper we prove that they are the complete set of solutions of (2.5). This is the content of the following two theorems:

Theorem 2.1. For the spin-S XXZ ferromagnetic chain with the anisotropic coupling $\Delta>1$, the following statements are valid: There are two translation invariant pure ground states, namely $\omega_{\uparrow}$ and $\omega_{\downarrow}$ determined by (2.6). Any pure infinite-volume ground state that is not translation invariant is either a kink, or an antikink ground state, belonging to the set described in [2].

Theorem 2.2. In the spin-S ferromagnetic Heisenberg chain with the isotropic coupling $\Delta=1$, all ground states (i.e., all solutions of (2.5)) are translation invariant. 
By combining these two theorems with previously known results, in particular [2], one can also prove that any pure infinite-volume ground state can be obtained as a thermodynamic limit of finite-volume states determined by eigenvectors of the finite-volume Hamiltonians with simple boundary terms. E.g., for $\Delta>1$, any pure infinite-volume ground state $\omega$ that is not translation invariant satisfies

$$
\mathrm{w}^{*}-\lim _{n \rightarrow \infty} \omega \circ \tau_{-n}=\omega_{\uparrow} \text { and } \mathrm{w}^{*}-\lim _{n \rightarrow \infty} \omega \circ \tau_{n}=\omega_{\downarrow},
$$

or

$$
\mathrm{w}^{*}-\lim _{n \rightarrow \infty} \omega \circ \tau_{-n}=\omega_{\downarrow} \text { and } \mathrm{w}^{*}-\lim _{n \rightarrow \infty} \omega \circ \tau_{n}=\omega_{\uparrow},
$$

where $\omega_{\uparrow}$ and $\omega_{\downarrow}$ are the states defined in (2.6). Here $\tau_{j}$ is the lattice translation by $j$ sites, i.e., $\tau_{j}(A) \in \mathcal{A}_{\Lambda+j}$, for all $A \in \mathcal{A}_{\Lambda}$. For any state $\omega, \omega \circ \tau_{j}$ is the state obtained by translation of $\omega$ over $j$ lattice spacings. In the first case (2.7), $\omega$ is the thermodynamic limit of finite-volume ground states of the local Hamiltonians $\tilde{H}_{\Lambda}^{+-}$defined in (3.4). In the second case (2.8), the same holds with the local Hamiltonians $\tilde{H}_{\Lambda}^{-+}$. For a more detailed version of Theorem 2.1 see Section 4.

Next, we give a brief outline of the proof of Theorem 2.1. The steps are formulated in a more general setting than just the XXZ chain, because this makes clear how one might generalize the result. In general, each step is a non-trivial problem, but in the case of the XXZ chain we have completed each of them. For a description of the proof of Theorem 2.2, which is closely related to the proof of Theorem 2.1, see Section 5.

Step 1. The first step is to determine the possible asymptotic behavior. This is relatively easy for one-dimensional systems. One only needs to determine the following sets of $\mathrm{w}^{*}$-limit points of arbitrary ground states:

$$
\begin{aligned}
& L P_{+}:=\mathrm{w}^{*} \text {-limit points of }\left\{\omega \circ \tau_{n} \mid \omega \text { satisfies }(2.5), \text { and } n \geq 0\right\} \\
& L P_{-}:=\mathrm{w}^{*} \text {-limit points of }\left\{\omega \circ \tau_{n} \mid \omega \text { satisfies }(2.5), \text { and } n \leq 0\right\} .
\end{aligned}
$$

In reflection symmetric models such as the XXZ chain, $L P_{-}=L P_{+}=: L P$, and we can, for simplicity, assume we are in this situation. In general, $L P$ is a convex set of states. For convenience, let us also assume it is a finitedimensional simplex consisting of translation invariant ground states of the model. In the case of the XXZ model with $\Delta>1$ this is the case and $L P$ is the segment $\left\{t \omega_{\uparrow}+(1-t) \omega_{\downarrow} \mid 0 \leq t \leq 1\right\}$. For the isotropic case $L P$ is also the set of translation invariant ground states, but the set of extreme points (the pure states) is infinite due to the continuous rotation symmetry. Everything below can be adapted to handle also this case. Denote by $L P_{0}$ the set of pure states in $L P$. 
Step 2. Next, construct orthogonal projections $P_{\xi} \in \mathcal{A}_{\mathrm{loc}}, \xi \in L P_{0}$, such that

$$
\xi\left(P_{\eta}\right) \approx \delta_{\xi, \eta}, \quad \text { for all } \xi, \eta \in L P_{0} .
$$

Such projections always exist on the basis of general abstract arguments [12], and one can assume that $\sum_{\xi} P_{\xi}=\mathbb{1}$.

Step 3. Then one shows that for all pairs of pure states $\xi, \eta \in L P_{0}$, the finite volume Hamiltonian can be written in the form

$$
\tilde{H}_{[-L, L]}^{(\xi, \eta)}=\sum_{x=-L-1}^{L} h_{x, x+1}^{(\xi, \eta)}+W_{L}^{(\xi, \eta)}
$$

for a suitable choice of the interaction $h_{x, x+1}^{(\xi, \eta)} \geq 0$ and boundary terms $W_{L}^{(\xi, \eta)}$, such that there is a ground state $\omega^{(\xi, \eta)}$ of the model with left and right asymptotics given by $\xi$ and $\eta$ respectively, and such that $\omega^{(\xi, \eta)}\left(h_{x, x+1}^{(\xi, \eta)}\right)=0$, for all $x \in \mathbb{Z}$, i.e., $\omega^{(\xi, \eta)}$ is a zero-energy ground state of the set of local Hamiltonians $\tilde{H}_{[-L, L]}^{(\xi, \eta)}$ in the sense of [2]. We assume that the complete set of zero-energy ground states of $\left\{\tilde{H}_{[-L, L]}^{(\xi, \eta)}\right\}$ is known, which is the case for the XXX and XXZ chains. For the XXZ chain the $\omega^{(\xi, \eta)}$ are the known ground states described above. The aim is to prove that any ground state $\omega$ in the sense of (2.5) is a convex combination of zero-energy ground states.

Step 4.. An arbitrary ground state can now be decomposed according to its left and right asymptotics, using the projections satisfying (2.11):

$$
\omega(\cdots) \approx \sum_{\xi, \eta \in L P_{0}} \omega\left(\tau_{-a}\left(P_{\xi}\right)(\cdots) \tau_{b}\left(P_{\eta}\right)\right), \quad a, b \gg 1
$$

Next, we use the following theorem due to Bratteli, Kishimoto, and Robinson [14].

Theorem 2.3. Let $\tilde{H}_{\Lambda}$ be a set of local Hamiltonians of the form $\tilde{H}_{\Lambda}=$ $H_{\Lambda \cup \partial \Lambda}+W_{\Lambda}$ where $W_{\Lambda} \in \mathcal{A}_{\Lambda^{c}}$ are arbitrary boundary terms, and $\partial \Lambda \subset \Lambda^{c}$. A state $\omega$ is a ground state in the sense of (2.5) iff $\omega$ satisfies

$$
\omega\left(\tilde{H}_{\Lambda}\right)=\inf \left\{\omega^{\prime}\left(\tilde{H}_{\Lambda}\right) \mid \omega^{\prime} \in C_{\Lambda}^{\omega}\right\}
$$

for all finite $\Lambda$, and where

$$
C_{\Lambda}^{\omega}:=\left\{\omega^{\prime}, \text { a state on } \mathcal{A} \mid \omega^{\prime}(A)=\omega(A) \text { for all } A \in \mathcal{A}_{\Lambda^{c}}\right\} .
$$


The difficult part is to construct suitable $\omega^{\prime} \in C_{\Lambda}^{\omega}$, and to prove an energy estimate for them. This is done by inserting a piece of the state $\omega^{(\xi, \eta)}$ into $\omega\left(\tau_{-a}\left(P_{\xi}\right)(\cdots) \tau_{b}\left(P_{\eta}\right)\right)$ appearing in the decomposition according to left and right asymptotics, and by using the appropriate boundary terms. By applying Theorem 2.3 , one then obtains that the states $\omega\left(\tau_{-a}\left(P_{\xi}\right)(\cdots) \tau_{b}\left(P_{\eta}\right)\right)$ satisfy

$$
\lim _{a, b \rightarrow \infty} \omega\left(\tau_{-a}\left(P_{\xi}\right) h_{x, x+1}^{(\xi, \eta)} \tau_{b}\left(P_{\eta}\right)\right)=0
$$

for all $x \in \mathbb{Z}$. In combination with Step 3 , it then follows that $\omega$ is a convex combination of zero-energy ground states.

From the outline above it is clear that the notion zero-energy ground states plays an important role in our completeness proof. We expect, however, that weaker notions can be substituted for it. E.g., the zero-energy condition is only used asymptotically, i.e., in the thermodynamic limit. A similar approach was reported by Matsui [15].

\section{Notations and Auxiliary Results}

\subsection{Notations}

We consider the standard ferromagnetic XXZ chain with arbitrary spin. The volumes $\Lambda$ we consider will always be finite unions of intervals $[a, b] \subset \mathbb{Z}$, and the half-infinite intervals of the form $[a,+\infty)$ and $(-\infty, b]$. The boundary of $\Lambda, \partial \Lambda$, is defined by

$$
\partial \Lambda=\{x \in \mathbb{Z} \backslash \Lambda \mid\{x-1, x+1\} \cap \Lambda \neq \emptyset\} .
$$

E.g., if $\Lambda=[a, b], \partial \Lambda=\{a-1, b+1\}$, and $\Lambda \cup \partial \Lambda=[a-1, b+1]$. The finite volume Hamiltonians of the XXZ Heisenberg chain are given by

$$
H_{\Lambda}=\sum_{x=a}^{b-1} h_{x, x+1}
$$

for all $a<b \in \mathbb{Z}$, and where

$$
h_{x, x+1}:=-\Delta^{-1}\left[S_{x}^{(1)} S_{x+1}^{(1)}+S_{x}^{(2)} S_{x+1}^{(2)}\right]-\left[S_{x}^{(3)} S_{x+1}^{(3)}-S^{2}\right], \text { for all } x \in \mathbb{Z} .
$$

Here $\Delta \geq 1$ is the anisotropic coupling, and $S=1 / 2,1,3 / 2, \ldots$ The normalization of the interactions (3.3) is such that one can consider the limit $\Delta \rightarrow \infty$ without difficulty. In this limit the models become the ferromagnetic classical Ising chains with spin- $S$. If $\Lambda_{1} \cap \Lambda_{2}=\emptyset, H_{\Lambda_{1} \cup \Lambda_{2}}=H_{\Lambda_{1}}+H_{\Lambda_{2}}$. 
This determines the Hamiltonians for all $\Lambda$ that are finite unions of finite intervals.

For the description of the kink and the antikink ground states we introduce two other Hamiltonians with specific boundary terms as follows: for any $\Lambda=[a, b]$,

$$
\tilde{H}_{\Lambda}^{+-}=H_{\Lambda \cup \partial \Lambda}+A(\Delta)\left[S_{b+1}^{(3)}-S_{a-1}^{(3)}\right]
$$

and

$$
\tilde{H}_{\Lambda}^{-+}=H_{\Lambda \cup \partial \Lambda}-A(\Delta)\left[S_{b+1}^{(3)}-S_{a-1}^{(3)}\right],
$$

where $A(\Delta):=S \sqrt{1-\Delta^{-2}}$. We will use the convention that tildes indicate that the local Hamiltonians include boundary terms. Typically, one has that $\tilde{H}_{\Lambda} \in \mathcal{A}_{\Lambda \cup \partial \Lambda}$, while $H_{\Lambda} \in \mathcal{A}_{\Lambda}$. The particular boundary terms in (3.4) and (3.5) make the quantum group $S U_{q}(2)$ symmetry that the XXZ chains possess explicit [16]. These are the local Hamiltonians studied in $[1,2,17]$. For convenience we also put

$$
\tilde{H}_{\Lambda}^{++}=\tilde{H}_{\Lambda}^{--}=H_{\Lambda \cup \partial \Lambda}
$$

We will also use the notation

$$
\begin{aligned}
h_{x, x+1}^{+-}= & -\Delta^{-1}\left[S_{x}^{(1)} S_{x+1}^{(1)}+S_{x}^{(2)} S_{x+1}^{(2)}\right] \\
& -\left[S_{x}^{(3)} S_{x+1}^{(3)}-S^{2}\right]+A(\Delta)\left[S_{x+1}^{(3)}-S_{x}^{(3)}\right], \\
h_{x, x+1}^{-+}=- & \Delta^{-1}\left[S_{x}^{(1)} S_{x+1}^{(1)}+S_{x}^{(2)} S_{x+1}^{(2)}\right] \\
& -\left[S_{x}^{(3)} S_{x+1}^{(3)}-S^{2}\right]-A(\Delta)\left[S_{x+1}^{(3)}-S_{x}^{(3)}\right],
\end{aligned}
$$

and

$$
h_{x, x+1}^{++}=h_{x, x+1}^{--}=h_{x, x+1} .
$$

Note that for $\alpha, \beta= \pm$, we have

$$
\tilde{H}_{[a, b]}^{\alpha \beta}=\sum_{x=a-1}^{b} h_{x, x+1}^{\alpha \beta} .
$$

In the case of $\alpha \beta=+-$ and $\alpha \beta=-+$, the boundary terms in corresponding Hamiltonians allow for ground states with a kink, or an antikink $[1,2]$.

\subsection{Properties of the ground states}

For the proof of Theorem 2.1 we will use detailed properties of the known ground states of the XXZ chain. In this section we bring together the properties known in the literature and prove some additional results that we need. 


\subsubsection{Finite-volume ground states}

Consider the spin-S XXZ ferromagnetic chain given by

$$
\tilde{H}_{\Lambda}^{\alpha \beta}=\sum_{x=a}^{b-1} h_{x, x+1}^{\alpha \beta}
$$

with the anisotropic coupling $\Delta>1$ and with $\Lambda \cup \partial \Lambda=[a, b]$, and where $h_{x, x+1}^{\alpha \beta}$ has been defined in $(3.7-3.9)$. For concreteness we consider the case $\alpha=-, \beta=+$. The case $\alpha=+, \beta=-$ is identical up to a reflection. The cases $\alpha=\beta=+$, and $\alpha=\beta=-$ are trivial. The ground state given by Alcaraz, Salinas and Wreszinski [1] is

$$
\Phi_{[a, b]}^{(M)}=\sum_{m_{x}} \prod_{x \in[a, b]} q^{x\left(S-m_{x}\right)} w\left(m_{x}\right)\left|\left\{m_{x}\right\}\right\rangle,
$$

where $m_{x}$ is the third component of the spin at the site $x$ with the range $-S \leq m_{x} \leq S$, and the sum is restricted to the configurations $\left\{m_{x}\right\}$ of the spins for which $\sum_{x} m_{x}=M$. The parameter $q$ is defined by $1<\Delta=$ $\left(q+q^{-1}\right) / 2$ with $0<q<1$, and the weights $w\left(m_{x}\right)$ are given by

$$
w(m)=\sqrt{\frac{(2 S) !}{(S-m) !(S+m) !}} .
$$

One can easily show that $h_{x, x+1}^{-+} \Phi_{[a, b]}^{(M)}=0$ for any $x \in[a, b-1]$. Thus we have $\tilde{H}_{\Lambda}^{-+} \Phi_{[a, b]}^{(M)}=0$. Since $h_{x, x+1}^{-+} \geq 0$, the vector $\Phi_{[a, b]}^{(M)}$ is a ground state of the Hamiltonian $\tilde{H}_{\Lambda}^{-+}$of (3.11). Further this ground state $\Phi_{[a, b]}^{(M)}$ is unique in the sector of the fixed magnetization $M$ from the Perron-Frobenius theorem.

Following [2], we introduce a vector

$$
\check{\Psi}_{[a, b]}^{-+}:=\sum_{M} \Phi_{[a, b]}^{(M)}=\bigotimes_{x \in[a, b]} \check{\chi}_{x}
$$

with

$$
\check{\chi}_{x}:=\sum_{m_{x}=-S}^{S} q^{x\left(S-m_{x}\right)} w\left(m_{x}\right)\left|m_{x}\right\rangle .
$$

The norm of $\check{\chi}_{x}$ can be easily computed as

$$
\left\|\check{\chi}_{x}\right\|=\left(1+q^{2 x}\right)^{S} .
$$

Therefore the normalized vector is

$$
\Psi_{[a, b]}^{-+}:=\bigotimes_{x \in[a, b]} \chi_{x}
$$


with

$$
\chi_{x}:=\frac{1}{\left(1+q^{2 x}\right)^{S}} \sum_{m_{x}=-S}^{S} q^{x\left(S-m_{x}\right)} w\left(m_{x}\right)\left|m_{x}\right\rangle
$$

We denote by $\psi_{[a, b]}^{-+}$the corresponding state, i.e., the expectation

$$
\psi_{[a, b]}^{-+}(\cdots)=\left\langle\Psi_{[a, b]}^{-+},(\cdots) \Psi_{[a, b]}^{-+}\right\rangle .
$$

Clearly this is an antikink with the center at the origin. Similarly we can construct the kink $\psi_{[a, b]}^{+-}$which is the reflection of the antikink centered at the origin.

The probability of finding $S_{x}^{(3)} \neq \alpha S$ at the site $x$ is given by

$$
\operatorname{Prob}_{[a, b]}^{-+}\left(S_{x}^{(3)} \neq \alpha S\right):=\psi_{[a, b]}^{-+}\left(11-P_{x}^{\alpha}\right), \quad \text { for } \alpha= \pm
$$

where $P_{x}^{ \pm}$are the projections onto the state with the spin $S_{x}^{(3)}=S$ at the site $x$ for the plus sign, and the state with $S_{x}^{(3)}=-S$ for the minus sign. Similarly we define by $\operatorname{Prob}_{[a, b]}^{+-}\left(S_{x}^{(3)} \neq \alpha S\right)$ the corresponding probabilities for the kink $\psi_{[a, b]}^{+-}$.

Lemma 3.1. The following estimates are valid:

$$
\begin{gathered}
\operatorname{Prob}_{[a, b]}^{-+}\left(S_{x}^{(3)} \neq \alpha S\right) \leq 2 S q^{2 \alpha x}, \quad \text { for } \alpha= \pm \\
\operatorname{Prob}_{[a, b]}^{+-}\left(S_{x}^{(3)} \neq \alpha S\right) \leq 2 S q^{-2 \alpha x}, \quad \text { for } \alpha= \pm .
\end{gathered}
$$

Proof. The probability of finding $S_{x}^{(3)}=S$ at the site $x$ is given by

$$
\operatorname{Prob}_{[a, b]}^{-+}\left(S_{x}^{(3)}=S\right):=\psi_{[a, b]}^{-+}\left(P_{x}^{+}\right)=\frac{1}{\left(1+q^{2 x}\right)^{2 S}}
$$

from (3.17), (3.18), and (3.19). Therefore

$$
\operatorname{Prob}_{[a, b]}^{-+}\left(S_{x}^{(3)} \neq S\right)=1-\frac{1}{\left(1+q^{2 x}\right)^{2 S}} \leq 1-e^{-2 S q^{2 x}} \leq 2 S q^{2 x}
$$

where we have used the following two inequalities: $1+u \leq e^{u}$ for $u \geq 0$, and $1-e^{-v} \leq v$ for $v \geq 0$. The rest are treated in the same way. 


\subsubsection{Infinite-volume ground states}

All pure ground states -at this point, we should rather say, all known pure ground states-, of the ferromagnetic XXZ chains happen to be zero-energy ground states, i.e., if $\omega$ is a pure ground state of a spin- $S$ ferromagnetic XXZ chain, then there is a choice of $\alpha, \beta= \pm$ such that

$$
\omega\left(h_{x, x+1}^{\alpha \beta}\right)=0, \quad \text { for all } x \in \mathbb{Z} .
$$

It is known from the work of Gottstein and Werner [2] (and some straightforward generalizations thereof) that the following list describes all zero-energy ground states of these models:

- If $\Delta=1$, all zero-energy ground states are translation invariant and the pure zero-energy ground states are all rotations of the state determined by

$$
\omega_{\uparrow}\left(S_{x}^{(3)}\right)=S, \quad \text { for all } x \in \mathbb{Z} .
$$

This means that there is $g \in \mathrm{SU}(2)$ such that

$$
\omega(A)=\omega_{\uparrow}\left(R_{g}(A)\right), \quad \text { for all } A \in \mathcal{A},
$$

where the rotation automorphism $R_{g}$ is determined by

$$
R_{g}(A)=\bigotimes_{x \in \Lambda} U_{x}^{*}(g) A U_{x}(g), \quad \text { for all } A \in \mathcal{A}_{\Lambda},
$$

where $U_{x}^{*}(g)$ is the $(2 S+1)$-dimensional irreducible unitary representation of $\mathrm{SU}(2)$ acting on the state space at site $x$. It is then clear that any zero-energy ground state of the isotropic chain is invariant under arbitrary permutations of the sites of the chain.

- If $\Delta>1$, as mentioned before, not all zero-energy ground states are translation invariant.

1. There are two translation invariant zero-energy ground states, $\omega_{\uparrow}$ and $\omega_{\downarrow}$, respectively determined by

$$
\omega_{\uparrow}\left(S_{x}^{(3)}\right)=S, \text { and } \omega_{\downarrow}\left(S_{x}^{(3)}\right)=-S, \quad \text { for all } x \in \mathbb{Z} .
$$

2. There is an infinite family of pure kink ground states, which are all obtained as thermodynamic limits of the finite-volume ground states of the Hamiltonians $\tilde{H}_{\Lambda}^{+-}$, described in the previous paragraphs. For a more detailed description of the resulting space of states see [2]. The kink ground states satisfy

$$
\omega\left(h_{x, x+1}^{+-}\right)=0 .
$$

Note that the translation invariant ground states have zero energy for each of the interactions $h_{x, x+1}^{\alpha \beta}$. 
3. There is an infinite family of pure antikink ground states which can be obtained from the kink ground states either by reflection (interchanging left and right), or by a rotation over $\pi$ about an axis in the XY plane (spin flip, interchanging up and down). All antikink ground states satisfy

$$
\omega\left(h_{x, x+1}^{-+}\right)=0 .
$$

Gottstein and Werner [2] also prove that if $\omega$ is a pure zero-energy ground state of the XXZ chains with local Hamiltonians $\tilde{H}_{\Lambda}^{-+}$, then exactly one of the following must be true:

- Either $\omega$ is translation invariant, and it is then either $\omega_{\uparrow}$ or $\omega_{\downarrow}$,

- or $\omega$ is represented by a unit vector in the GNS Hilbert space of the state $\omega_{0}$ obtained by the following thermodynamic limit:

$$
\omega_{0}(A)=\lim _{b \rightarrow+\infty} \lim _{a \rightarrow-\infty} \psi_{[a, b]}^{-+}(A)
$$

which describes an antikink centered at the origin. Here $\psi_{[a, b]}^{-+}$is given by (3.19). For a description of the GNS representation $\pi_{\omega_{0}}$ of $\omega_{0}$, and the GNS Hilbert space $\mathcal{H}_{\omega_{0}}$, see [2] or [17]. So, in this case there is a vector $\psi \in \mathcal{H}_{\omega_{0}}$, such that for all $A \in \mathcal{A}$

$$
\omega(A)=\left\langle\psi, \pi_{\omega_{0}}(A) \psi\right\rangle .
$$

Moreover, $\psi$ belongs to the kernel of the GNS Hamiltonian. Together with Theorem 4.4, this result (and its analogue for kink states) also proves that all infinite-volume ground states of the XXZ chains are thermodynamic limits of finite-volume ground states of local Hamiltonians with one-site boundary terms as, e.g., the $\tilde{H}_{\Lambda}^{\alpha \beta}$ defined in (3.5-3.6).

\section{Proof of Theorem 2.1}

We begin with three short lemmas that will be used in the proof of Theorem 2.1 and Theorem 2.2 .

Lemma 4.1. Let $\tilde{H}_{[a, b]}=\sum_{x=a-1}^{b} h_{x, x+1}$ be local Hamiltonians with a translation invariant interaction satisfying $0 \leq h_{x, x+1} \leq h \mathbb{1}$, and suppose there exists a state $\eta$ of $\mathcal{A}$ such that $\eta\left(h_{x, x+1}\right)=0$, for all $x \in \mathbb{Z}$. Then, if $\omega$ is a ground state, i.e., $\omega$ satisfies (2.5), we have

$$
0 \leq \omega\left(\tilde{H}_{[a, b]}\right) \leq 2 h
$$

for all $a<b \in \mathbb{Z}$, and

$$
\lim _{x \rightarrow \pm \infty} \omega\left(h_{x, x+1}\right)=0 .
$$


Proof. Since $h_{x, x+1} \geq 0$, we get the lower bound. To obtain the upper bound, we consider the trial state

$$
\omega^{\prime}=\omega_{[a, b]^{c}} \otimes \eta_{[a, b]},
$$

where the subscripts denote the restriction to the algebra on the corresponding subvolume. Then, by Theorem 2.3 , we have

$$
\omega\left(\tilde{H}_{[a, b]}\right) \leq \omega^{\prime}\left(\tilde{H}_{[a, b]}\right)=\left(\omega_{[a, b] c} \otimes \eta_{[a, b]}\right)\left(h_{a-1, a}+h_{b, b+1}\right) \leq 2 h .
$$

This proves (4.1). From (4.1) it follows that $\lim \omega\left(\tilde{H}_{[a, b]}\right)$ is a convergent sum of non-negative terms terms and, hence, (4.2) is obvious.

Lemma 4.2. Suppose $a_{n} \in \mathbb{Z}$ such that either $\lim _{n} a_{n}=+\infty$ or $\lim _{n} a_{n}=$ $-\infty$, and suppose $\omega$ is a ground state of the spin $-S X X Z$ chain with $\Delta>1$. If there exists

$$
\lim _{n} \omega \circ \tau_{a_{n}}=\eta,
$$

then there exists $t \in[0,1]$ such that

$$
\eta=t \omega_{\uparrow}+(1-t) \omega_{\downarrow}
$$

where $\omega_{\uparrow}$ and $\omega_{\downarrow}$ are the "up" and "down" ground states defined in (2.6).

Proof. By Lemma 4.1 we have that $\eta$ is a zero-energy ground state with

$$
\eta\left(h_{x, x+1}\right)=0, \text { for all } x \in \mathbb{Z} .
$$

This implies that $\eta$ is a convex combination of the two translation invariant zero-energy ground states $\omega_{\uparrow}$ and $\omega_{\downarrow}$.

We will use the following elementary lemma in our estimates.

Lemma 4.3. Let $\omega$ be a state of a $C^{*}$-algebra $\mathcal{A}$, and suppose $P \in \mathcal{A}$ is an orthogonal projection. Then, for all $A \in \mathcal{A}$ we have

$$
|\omega(A)-\omega(P A P)| \leq 2 \sqrt{1-\omega(P)}\|A\| .
$$

Proof. Note that

$$
\omega(A)-\omega(P A P)=\omega(A(\mathbb{1}-P))+\omega((\mathbb{1}-P) A P) .
$$

The two terms in the right-hand side can be estimated using the CauchySchwarz inequality for the positive sesqui-linear form $(X, Y) \mapsto \omega\left(X^{*} Y\right)$ :

$$
\begin{aligned}
|\omega(A(\mathbb{1}-P))|^{2} & \leq \omega(\mathbb{1}-P) \omega\left(A A^{*}\right), \\
|\omega((\mathbb{1}-P) A P)|^{2} & \leq \omega(\mathbb{1}-P) \omega\left(P A A^{*} P\right) .
\end{aligned}
$$

As $\omega\left(A A^{*}\right)$ and $\omega\left(P A A^{*} P\right)$ are both bounded by $\|A\|^{2}$, the result follows. 
The set of all states of $\mathcal{A}$ is w*-compact. Therefore, for any state $\omega$ there are sequences $a_{n}$ of integers, with $\lim _{n} a_{n}=+\infty$, and such that the $\mathrm{w} *$ limits of $\omega \circ \tau_{a_{n}}$ and $\omega \circ \tau_{-a_{n}}$ exist. The following theorem is then sufficient to prove Theorem 2.1 .

Theorem 4.4. Let $\omega$ be any ground state of the spin-S XXZ ferromagnetic chain with anisotropic coupling $\Delta>1$, and let $\left[a_{n}, b_{n}\right]$ be a sequence of intervals tending to $\mathbb{Z}$ (i.e., $a_{n} \rightarrow-\infty$ and $b_{n} \rightarrow+\infty$ ), such that

$$
\mathrm{w}^{*}-\lim _{n \rightarrow \infty} \omega \circ \tau_{-a_{n}} \equiv \omega_{-\infty}=t_{-\infty} \omega_{\uparrow}+\left(1-t_{-\infty}\right) \omega_{\downarrow}
$$

and

$$
\mathrm{w}^{*}-\lim _{n \rightarrow \infty} \omega \circ \tau_{b_{n}} \equiv \omega_{+\infty}=t_{+\infty} \omega_{\uparrow}+\left(1-t_{+\infty}\right) \omega_{\downarrow} .
$$

Here $t_{ \pm \infty} \in[0,1]$. Then the following properties hold:

i) $\omega$ has well-defined asymptotics, i.e.,

$$
w^{*}-\lim _{n \rightarrow \infty} \omega \circ \tau_{ \pm n}=\omega_{\mp \infty}
$$

ii) $\omega$ has a convex decomposition as follows

$$
\omega=t^{++} \omega_{\uparrow}+t^{--} \omega_{\downarrow}+t^{+-} \varphi^{+-}+t^{-+} \varphi^{-+},
$$

where the states $\varphi^{+-}$and $\varphi^{-+}$satisfy, for all $x \in \mathbb{Z}$,

$$
\varphi^{+-}\left(h_{x, x+1}^{+-}\right)=0, \quad \varphi^{-+}\left(h_{x, x+1}^{-+}\right)=0 .
$$

Note that the complete list of such states $\varphi^{+-}$and $\varphi^{-+}$is known due to the work of Gottstein and Werner described in the previous section. The state $\varphi^{+-}$is a convex combination of the translations of a kink state, and $\varphi^{-+}$is a convex combination of the translations of an antikink state. The following relations between the convex combination coefficients are obvious:

$$
t_{-\infty}=t^{++}+t^{+-}, \quad t_{+\infty}=t^{-+}+t^{++} .
$$

Proof. We use the Bratteli-Kishimoto-Robinson characterization of ground states stated in Theorem 2.3. A suitable choice for the boundary terms is:

$$
W_{[a, b]}=-2 S A(\Delta)\left(P_{a-1}^{+} P_{b+1}^{-}+P_{a-1}^{-} P_{b+1}^{+}\right),
$$

where $P_{x}^{ \pm}$are the projections onto the state with $S_{x}^{(3)}=S$ at the site $x$ for the plus sign, and the state with $S_{x}^{(3)}=-S$ for the minus sign. The corresponding local Hamiltonian is

$$
\tilde{H}_{[a, b]}=\sum_{x=a-1}^{b} h_{x, x+1}+W_{[a, b]} .
$$


First, we will show that for any ground state $\omega$ satisfying the assumptions of the theorem the quantity $E_{\Lambda}(\omega)$ defined by

$$
E_{\Lambda}(\omega)=\inf \left\{\omega^{\prime}\left(\tilde{H}_{\Lambda}\right) \mid \omega_{\Lambda^{c}}^{\prime}=\omega_{\Lambda^{c}}\right\}
$$

tends to zero as $\Lambda=\left[a_{n}, b_{n}\right] \uparrow \mathbb{Z}$ by showing that $\liminf _{n} E_{\left[a_{n}, b_{n}\right]}(\omega) \geq$ 0 (part a), and $\lim \sup _{n} E_{\left[a_{n}, b_{n}\right]}(\omega) \leq 0$ (part b). This fact can then be combined with the assumed asymptotics $(4.12-4.13)$ to complete the proof of the theorem (see part c).

We write

a) Proof of $\liminf _{n} E_{\left[a_{n}, b_{n}\right]}(\omega) \geq 0$.

$$
P_{x}=P_{x}^{+}+P_{x}^{-} .
$$

The assumed asymptotic behavior of $\omega$ implies that for any $\epsilon>0$, there exists $N$ such that, for all $n \geq N$, we have

$$
\begin{aligned}
\omega\left(\mathbb{1}-P_{a_{n}-1}\right) & \leq \epsilon, \\
\omega\left(P_{a_{n}-1}^{\alpha}\left(\mathbb{1}-P_{a_{n}}^{\alpha}\right)\right) & \leq \epsilon, \\
\omega\left(P_{a_{n}-1}^{\alpha} A_{a_{n}-1} P_{a_{n}-1}^{\beta}\right) & \leq \epsilon, \quad \text { if } \alpha \neq \beta \quad \text { for any } A_{a_{n}-1} \in \mathcal{A}_{\left\{a_{n}-1\right\}}
\end{aligned}
$$

and similar inequalities hold with $a_{n}-1$ replaced by $b_{n}+1$. Clearly, for $\Lambda=\left[a_{n}, b_{n}\right], n \geq N$, any $\omega^{\prime}$ such that $\omega_{\Lambda^{c}}^{\prime}=\omega_{\Lambda^{c}}$ satisfies the same inequalities. In order to estimate the finite-volume energy of such $\omega^{\prime}$, first introduce resolutions of the identity at the site $a_{n}-1$ as follows

$$
\begin{aligned}
& \omega^{\prime}\left(\tilde{H}_{\left[a_{n}, b_{n}\right]}\right)=\omega^{\prime}\left(P_{a_{n}-1} \tilde{H}_{\left[a_{n}, b_{n}\right]} P_{a_{n}-1}\right) \\
& +\omega^{\prime}\left(P_{a_{n}-1} \tilde{H}_{\left[a_{n}, b_{n}\right]}\left(\mathbb{1}-P_{a_{n}-1}\right)\right)+\omega^{\prime}\left(\left(\mathbb{1}-P_{a_{n}-1}\right) \tilde{H}_{\left[a_{n}, b_{n}\right]} P_{a_{n}-1}\right) \\
& +\omega^{\prime}\left(\left(\mathbb{1}-P_{a_{n}-1}\right) \tilde{H}_{\left[a_{n}, b_{n}\right]}\left(\mathbb{1}-P_{a_{n}-1}\right)\right) .
\end{aligned}
$$

From (4.22), the non-vanishing terms are the first and the fourth ones. The fourth term is non-negative. In fact it is written as

$$
\begin{aligned}
& \omega^{\prime}\left(\left(\mathbb{1}-P_{a_{n}-1}\right) \tilde{H}_{\left[a_{n}, b_{n}\right]}\left(\mathbb{1}-P_{a_{n}-1}\right)\right)= \\
& \sum_{x=a_{n}-1}^{b_{n}} \omega^{\prime}\left(\left(\mathbb{1}-P_{a_{n}-1}\right) h_{x, x+1}\left(\mathbb{1}-P_{a_{n}-1}\right)\right) \geq 0
\end{aligned}
$$

because the contribution from the boundary term $W_{\left[a_{n}, b_{n}\right]}$ of (4.18) is vanishing. We decompose the first term in the right-hand side of (4.25) as

$$
\begin{aligned}
& \omega^{\prime}\left(P_{a_{n}-1} \tilde{H}_{\left[a_{n}, b_{n}\right]} P_{a_{n}-1}\right)=\omega^{\prime}\left(P_{a_{n}-1}^{+} \tilde{H}_{\left[a_{n}, b_{n}\right]} P_{a_{n}-1}^{+}\right) \\
& +\omega^{\prime}\left(P_{a_{n}-1}^{+} \tilde{H}_{\left[a_{n}, b_{n}\right]} P_{a_{n}-1}^{-}\right)+\omega^{\prime}\left(P_{a_{n}-1}^{-} \tilde{H}_{\left[a_{n}, b_{n}\right]} P_{a_{n}-1}^{+}\right) \\
& +\omega^{\prime}\left(P_{a_{n}-1}^{-} \tilde{H}_{\left[a_{n}, b_{n}\right]} P_{a_{n}-1}^{-}\right) .
\end{aligned}
$$


From (4.24), the only non-vanishing terms are of the form $\omega^{\prime}\left(P_{a_{n}-1}^{\alpha} \tilde{H}_{\left[a_{n}, b_{n}\right]} P_{a_{n}-1}^{\alpha}\right)$. Since similar properties hold for the site $b_{n}+1$, we have only to treat the forms

$$
\omega^{\prime}\left(P_{a_{n}-1}^{\alpha} P_{b_{n}+1}^{\beta} \tilde{H}_{\left[a_{n}, b_{n}\right]} P_{a_{n}-1}^{\alpha} P_{b_{n}+1}^{\beta}\right)
$$

as the rest of non-vanishing contributions. We show that these are nonnegative. To do this we consider the following decompositions of the Hamiltonian:

$$
\tilde{H}_{[a, b]}=\tilde{H}_{[a, b]}^{\alpha \beta}+\delta W_{[a, b]}^{\alpha \beta}
$$

with

$$
\delta W_{[a, b]}^{\alpha \beta}:=A(\Delta)\left[\frac{1}{2}(\alpha-\beta)\left(S_{a-1}^{(3)}-S_{b+1}^{(3)}\right)-2 S\left(P_{a-1}^{+} P_{b+1}^{-}+P_{a-1}^{-} P_{b+1}^{+}\right)\right] .
$$

Using this expression, one can easily show

$$
P_{a-1}^{\alpha} P_{b+1}^{\beta} \tilde{H}_{[a, b]} P_{a-1}^{\alpha} P_{b+1}^{\beta}=P_{a-1}^{\alpha} P_{b+1}^{\beta} \tilde{H}_{[a, b]}^{\alpha \beta} P_{a-1}^{\alpha} P_{b+1}^{\beta} \geq 0
$$

Clearly the corresponding terms in the expectation are non-negative. Thus, we have shown that

$$
\omega\left(\tilde{H}_{\left[a_{n}, b_{n}\right]}\right) \geq-C \times \epsilon
$$

where $C$ is a positive constant and as $\epsilon>0$ is arbitrary, this proves $\liminf { }_{n} E_{\left[a_{n}, b_{n}\right]}(\omega) \geq 0$.

b) Proof of $\limsup \sup _{n} E_{\left[a_{n}, b_{n}\right]}(\omega) \leq 0$.

In order to obtain an upper bound implying that also $\lim \sup E_{\Lambda}(\omega)=0$, we choose a trial state $\omega^{\prime}$ defined as follows:

$$
\omega^{\prime}=\sum_{\alpha, \beta= \pm} \xi^{\alpha \beta}+\xi^{\prime}
$$

with

$$
\xi^{\alpha \beta}=\psi_{\left[a_{n}+1, b_{n}-1\right]}^{\alpha \beta} \otimes \omega_{\left[a_{n}+1, b_{n}-1\right]^{c}}\left(P_{a_{n}}^{\alpha} P_{b_{n}}^{\beta}(\cdots) P_{a_{n}}^{\alpha} P_{b_{n}}^{\beta}\right)
$$

and

$$
\xi^{\prime}=\psi_{\left[a_{n}+1, b_{n}-1\right]}^{++} \otimes \omega_{\left[a_{n}+1, b_{n}-1\right]^{c}}\left(\left(\mathbb{1}-P_{a_{n}} P_{b_{n}}\right)(\cdots)\left(\mathbb{1}-P_{a_{n}} P_{b_{n}}\right)\right),
$$

where $\psi_{\left[a_{n}+1, b_{n}-1\right]}^{-+}$is given by (3.19), i.e., the antikink centered at the origin, $\psi_{\left[a_{n}+1, b_{n}-1\right]}^{+-}$is the kink which is the reflection of the kink, and $\psi^{\alpha \alpha}$ is the state with all spins $S_{x}^{(3)}=\alpha S$ for $\alpha= \pm$. 
Before making the energy estimates, we check that $\omega^{\prime}(A)=\omega(A)$ for all $A \in \mathcal{A}_{\left[a_{n}, b_{n}\right]^{c}}$. This is verified as follows:

$$
\begin{aligned}
\omega^{\prime}(A) & =\sum_{\alpha, \beta= \pm} \xi^{\alpha \beta}(A)+\xi^{\prime}(A) \\
& =\sum_{\alpha, \beta= \pm} \omega\left(P_{a_{n}}^{\alpha} P_{b_{n}}^{\beta} A\right)+\omega\left(\left(\mathbb{1}-P_{a_{n}} P_{b_{n}}\right) A\right) \\
& =\omega\left(P_{a_{n}} P_{b_{n}} A\right)+\omega\left(\left(\mathbb{1}-P_{a_{n}} P_{b_{n}}\right) A\right)=\omega(A)
\end{aligned}
$$

for $A \in \mathcal{A}_{\left[a_{n}, b_{n}\right]^{c}}$.

To compute the energy of each term we again use the decomposition (4.29). The interior terms of the Hamiltonian, i.e., $\tilde{H}_{\left[a_{n}+2, b_{n}-2\right]}^{\alpha \beta}$, are identically zero. Therefore we have

$$
\begin{array}{r}
\xi^{\alpha \beta}\left(\tilde{H}_{\left[a_{n}, b_{n}\right]}\right)=\xi^{\alpha \beta}\left(h_{a_{n}-1, a_{n}}^{\alpha \beta}+h_{a_{n}, a_{n}+1}^{\alpha \beta}+h_{b_{n}-1, b_{n}}^{\alpha \beta}+h_{b_{n}, b_{n}+1}^{\alpha \beta}\right) \\
+\xi^{\alpha \beta}\left(\delta W_{\left[a_{n}, b_{n}\right]}^{\alpha \beta}\right)
\end{array}
$$

and

$$
\begin{array}{r}
\xi^{\prime}\left(\tilde{H}_{\left[a_{n}, b_{n}\right]}\right)=\xi^{\prime}\left(h_{a_{n}-1, a_{n}}+h_{a_{n}, a_{n}+1}+h_{b_{n}-1, b_{n}}+h_{b_{n}, b_{n}+1}\right) \\
-2 S A(\Delta) \xi^{\prime}\left(P_{a_{n}-1}^{+} P_{b_{n}+1}^{-}+P_{a_{n}-1}^{-} P_{b_{n}+1}^{+}\right) .
\end{array}
$$

Owing to (4.22) and

$$
\mathbb{1}-P_{a_{n}} P_{b_{n}}=\left(\mathbb{1}-P_{a_{n}}\right) P_{b_{n}}+P_{a_{n}}\left(1-P_{b_{n}}\right)+\left(\mathbb{1}-P_{a_{n}}\right)\left(\mathbb{1}-P_{b_{n}}\right),
$$

the right-hand side of (4.39) tends to zero as $n \rightarrow \infty$.

By definition, we can write $\xi^{\alpha \beta}\left(\tilde{H}_{\left[a_{n}, b_{n}\right]}\right)$ of $(4.38)$ as

$$
\begin{aligned}
\xi^{\alpha \beta}\left(\tilde{H}_{\left[a_{n}, b_{n}\right]}\right) & =\omega\left(P_{b_{n}}^{\beta} P_{a_{n}}^{\alpha} h_{a_{n}-1, a_{n}}^{\alpha \beta} P_{a_{n}}^{\alpha}\right)+\xi^{\alpha \beta}\left(h_{a_{n}, a_{n}+1}^{\alpha \beta}\right)+\xi^{\alpha \beta}\left(h_{b_{n}-1, b_{n}}^{\alpha \beta}\right) \\
& +\omega\left(P_{a_{n}}^{\alpha} P_{b_{n}}^{\beta} h_{b_{n}-1, b_{n}}^{\alpha \beta} P_{b_{n}}^{\beta}\right)+\omega\left(P_{a_{n}}^{\alpha} P_{b_{n}}^{\beta} \delta W_{\left[a_{n}, b_{n}\right]}^{\alpha \beta}\right)
\end{aligned}
$$

The first term in the right-hand side is written as

$$
\begin{array}{r}
\omega\left(P_{b_{n}}^{\beta} P_{a_{n}}^{\alpha} h_{a_{n}-1, a_{n}}^{\alpha \beta} P_{a_{n}}^{\alpha}\right)=\omega\left(P_{b_{n}}^{\beta} P_{a_{n}}^{\alpha} h_{a_{n}-1, a_{n}}^{\alpha \beta} P_{a_{n}}^{\alpha}\left(\mathbb{1}-P_{a_{n}-1}^{\alpha}\right)\right) \\
+\omega\left(P_{b_{n}}^{\beta} P_{a_{n}}^{\alpha} h_{a_{n}-1, a_{n}}^{\alpha \beta} P_{a_{n}}^{\alpha} P_{a_{n}-1}^{\alpha}\right) .
\end{array}
$$

This first term in the right-hand side is vanishing as $a_{n} \rightarrow-\infty$ from (4.23), and the second term also is vanishing because $h_{a_{n}-1, a_{n}}^{\alpha \beta} P_{a_{n}}^{\alpha} P_{a_{n}-1}^{\alpha}=0$. Therefore the first term $\omega\left(P_{b_{n}}^{\beta} P_{a_{n}}^{\alpha} h_{a_{n}-1, a_{n}}^{\alpha \beta} P_{a_{n}}^{\alpha}\right)$ of (4.41) is vanishing. Similarly the fourth term in the right-hand side of (4.41) is vanishing as $b_{n} \rightarrow+\infty$. 
The fifth term in the right-hand side of (4.41) tends to zero as $n \rightarrow \infty$. To show this, we introduce a resolution of the identity

$$
\begin{aligned}
\mathbb{1}=P_{a_{n}-1}^{\alpha} P_{b_{n}+1}^{\beta}+P_{a_{n}-1}^{\alpha}\left(11-P_{b_{n}+1}^{\beta}\right)+ & \left(1-P_{a_{n}-1}^{\alpha}\right) P_{b_{n}+1}^{\beta} \\
& +\left(1-P_{a_{n}-1}^{\alpha}\right)\left(11-P_{b_{n}+1}^{\beta}\right) .
\end{aligned}
$$

Owing to this and (4.23), we have only to show that

$$
\omega\left(P_{a_{n}}^{\alpha} P_{b_{n}}^{\beta} \delta W_{\left[a_{n}, b_{n}\right]}^{\alpha \beta} P_{a_{n}-1}^{\alpha} P_{b_{n}+1}^{\beta}\right)
$$

is vanishing in the limit $n \rightarrow \infty$. But this is identically zero for all $\alpha, \beta= \pm$.

The rest are the second and the third terms in the right-hand side of (4.41). We treat only the second term because the third one is treated in the same way. To begin with, we note the following: From Lemma 3.1 and Lemma 4.3 we obtain

$$
\begin{array}{r}
\left\|\psi_{\left[a_{n}+1, b_{n}-1\right]}^{\alpha \beta}\left(P_{a_{n}+1}^{\alpha} P_{b_{n}-1}^{\beta}(\cdots) P_{a_{n}+1}^{\alpha} P_{b_{n}-1}^{\beta}\right)-\psi_{\left[a_{n}+1, b_{n}-1\right]}^{\alpha \beta}(\cdots)\right\| \\
\leq \mu \exp \left[-\nu \min \left\{-a_{n}, b_{n}\right\}\right]
\end{array}
$$

for some positive constants $\mu, \nu$, and $\min \left\{-a_{n}, b_{n}\right\}$ represents the distance of the position of the center of the kink to the origin. Combining (4.45) with

$$
P_{a_{n}}^{\alpha} P_{a_{n}+1}^{\alpha} h_{a_{n}, a_{n}+1}^{\alpha \beta} P_{a_{n}}^{\alpha} P_{a_{n}+1}^{\alpha}=0 \quad \text { for all } \alpha, \beta= \pm
$$

we get the desired result $\xi^{\alpha \beta}\left(h_{a_{n}, a_{n}+1}^{\alpha \beta}\right) \rightarrow 0$ as $a_{n} \rightarrow-\infty$. This concludes the proof of $\limsup _{n} E_{\left[a_{n}, b_{n}\right]}(\omega) \leq 0$.

c) We can now complete the proof as follows. From the asymptotics it follows that there is a sequence $\epsilon_{n} \downarrow 0$ such that

$$
\left|\omega_{\left[a_{n}, b_{n}\right]}(A)-\sum_{\alpha, \beta= \pm} \omega\left(P_{a_{n}-1}^{\alpha} P_{b_{n}+1}^{\beta} A\right)\right| \leq \epsilon_{n}\|A\|
$$

for all $A \in \mathcal{A}_{\left[a_{n}, b_{n}\right]}$. This implies that there are four subsequences of states defined by

$$
\omega_{k}^{\alpha \beta}(A)=\frac{\omega\left(P_{a_{n_{k}}-1}^{\alpha} P_{b_{n_{k}}+1}^{\beta} A P_{a_{n_{k}}-1}^{\alpha} P_{b_{n_{k}}+1}^{\beta}\right)}{\omega\left(P_{a_{n_{k}}-1}^{\alpha} P_{b_{n_{k}}+1}^{\beta}\right)}
$$

with the following properties:

$$
\begin{aligned}
& w^{*}-\lim _{k \rightarrow \infty} \omega_{k}^{\alpha \beta} \equiv \omega^{\alpha \beta} \text { exists, } \\
& w^{*}-\lim _{k \rightarrow \infty}\left|\omega-\sum_{\alpha, \beta= \pm} t^{\alpha \beta} \omega_{k}^{\alpha \beta}\right|=0, \quad \text { with } t^{\alpha \beta} \geq 0 \\
& \lim _{k \rightarrow \infty} \omega_{k}^{\alpha \beta}\left(h_{x, x+1}^{\alpha \beta}\right)=0 \quad \text { for all } x \in \mathbb{Z}
\end{aligned}
$$


The last property (4.51) follows from the above results in parts a and $\mathrm{b}$. To see this, we note that

$$
\omega_{k}^{\alpha \beta}\left(\tilde{H}_{\left[a_{n_{k}}, b_{n_{k}}\right]}\right)=\omega_{k}^{\alpha \beta}\left(\tilde{H}_{\left[a_{n_{k}}, b_{n_{k}}\right]}^{\alpha \beta}\right)+\omega_{k}^{\alpha \beta}\left(\delta W_{\left[a_{n_{k}}, b_{n_{k}}\right]}^{\alpha \beta}\right),
$$

where we have used the decompositions (4.29) of the Hamiltonian $\tilde{H}_{[a, b]}$. The second term in the right-hand side is vanishing as $a_{n_{k}} \rightarrow-\infty, b_{n_{k}} \rightarrow$ $+\infty$ from the asymptotics and the definition (4.30) of $\delta W_{[a, b]}^{\alpha \beta}$. Combining these observations with (4.50) and with the results of parts a and b, i.e., $\omega\left(\tilde{H}_{\left[a_{n}, b_{n}\right]}\right) \rightarrow 0$ as $a_{n} \rightarrow-\infty, b_{n} \rightarrow+\infty$, we have

$$
\omega_{k}^{\alpha \beta}\left(\tilde{H}_{\left[a_{n_{k}}, b_{n_{k}}\right]}^{\alpha \beta}\right)=\sum_{x=a_{n_{k}}-1}^{b_{n_{k}}} \omega_{k}^{\alpha \beta}\left(h_{x, x+1}^{\alpha \beta}\right) \rightarrow 0 \quad \text { as } a_{n_{k}} \rightarrow-\infty, b_{n_{k}} \rightarrow+\infty .
$$

This implies (4.51) because $h_{x, x+1}^{\alpha \beta} \geq 0$ for all $\alpha, \beta= \pm$ and for all $x \in \mathbb{Z}$.

As the convex decomposition (4.15), if it exists, is unique, its terms must be proportional to the $\omega^{\alpha \beta}$ because of (4.50). The property (4.51) identifies the $\omega^{\alpha \beta}$ as the known zero-energy states, which proves the statement ii) of Theorem 4.4. The statement i) follows from ii).

\section{$5 \quad$ Proof of Theorem 2.2}

The proof of the absence of non-translation-invariant ground states in the isotropic case is similar to the proof in the anisotropic case (Theorem 4.4). It differs from it in two points. The first difference is that in the isotropic case all ground states turn out to be zero energy ground states for the same local Hamiltonians. This simplifies the proof. The second difference, however, makes the proof more subtle than in the anisotropic case. This is due to the broken continuous rotation symmetry. The possible asymptotics of pure ground states now depend on two continuous parameters, which can be taken to be two angles: $(\theta, \phi)=\Omega \in S^{2}$. For the same reason the excitation spectrum is gapless and, therefore, the method of proof followed in [9] cannot be adapted to the isotropic case.

The state of a single spin pointing in the direction $\Omega$ is represented by the vector

$$
|\Omega\rangle:=U(\Omega)|S\rangle
$$

where

$$
U(\Omega):=e^{-i \phi S^{(3)}} e^{-i \theta S^{(2)}}
$$


and $|S\rangle$ is the normalized eigenvector of $S^{(3)}$ satisfying $S^{(3)}|S\rangle^{-}=S|S\rangle$. It follows that the vectors

$$
U(\Omega)|S\rangle, \quad \Omega \in S^{2}
$$

span the $(2 S+1)$-dimensional irreducible unitary representation of $\mathrm{SU}(2)$. For the same reason the vectors

$$
|\Omega\rangle_{\Lambda}:=\bigotimes_{x \in \Lambda}(|\Omega\rangle)_{x}
$$

span the maximum total spin subspace for any finite volume $\Lambda \subset \mathbb{Z}$. It is also straightforward to check that the orthogonal projection onto the subspace of maximal total spin, $P_{\Lambda}$, can be written as [18]

$$
P_{\Lambda}:=\frac{2 k S+1}{4 \pi} \int d \Omega Q_{\Lambda}(\Omega)
$$

where, as usual, $d \Omega=\sin \theta d \theta d \phi$, and

$$
Q_{\Lambda}(\Omega)=\bigotimes_{x \in \Lambda}(|\Omega\rangle\langle\Omega|)_{x}
$$

It is obvious from the form of the isotropic Hamiltonian ((3.6) with $\Delta=1$ ) that zero-energy states are supported by the maximum total spin subspace on each finite volume, which is permutation invariant. It follows immediately that all zero-energy ground states of this Hamiltonian are translation invariant. Therefore, for the proof of Theorem 2.2 it is sufficient to show that all ground states are zero-energy states for this Hamiltonian. By Theorem 2.3, and the fact that the local interactions are non-negative, this will follow if we prove that for any ground state

$$
\lim _{\Lambda \uparrow \mathbb{Z}} E_{\Lambda}(\omega)=0
$$

where $E_{\Lambda}(\omega)$ is defined in (4.20). By Lemma 4.1 we have to do this for any $\omega$ with left and right asymptotics such that

$$
\lim _{x \rightarrow \pm \infty} \omega\left(\mathbb{1}-P_{\Lambda+x}\right)=0
$$

for all finite $\Lambda \subset \mathbb{Z}$. To do this we follow the same strategy as in the proof of Theorem 2.1: we need to construct local modifications of an arbitrary ground state $\omega$ that have arbitrarily low energy. This will be done by inserting a long-wavelength spin-wave state that gradually turns the spin from $\Omega^{-}$ to $\Omega^{+}$, conditioned upon the asymptotic orientation to the left and to the right being $\Omega^{-}$and $\Omega^{+}$respectively. The sum over the possible asymptotic 
behaviors appearing in the proof of Theorem 2.1 will here become an integral over $\Omega^{-}$and $\Omega^{+}$. Some care has to be taken with the conditioning in order not to spoil the energy estimates, which need to be done carefully, too. A quick estimate produces useless bounds. Now, we fill in the technical details of the proof sketched above.

\section{Proof of Theorem 2.2:}

As explained above we want to show (5.7). Due to the non-negative interaction we only need to show

$$
\underset{\Lambda \uparrow \mathbb{Z}}{\limsup } E_{\Lambda}(\omega) \leq 0
$$

Let $\omega$ be a ground state. We will construct a trial state $\omega^{\prime}$ which coincides with $\omega$ outside a finite interval $\Lambda=[a, b]$. We will use a corridor of $m$ sites to perform the conditioning. Therefore, let $m \geq 1$ be such that $b-a+1>2 m$. The state $\omega^{\prime}$ is then defined by

$$
\omega^{\prime}:=\int d \Omega^{+} \int d \Omega^{-} \eta_{[a+m, b-m]}^{\Omega^{+}, \Omega^{-}} \otimes \omega_{[a+m, b-m]^{c}}^{\Omega^{+}, \Omega^{-}}+\omega^{\prime \prime},
$$

where $\eta_{[a+m, b-m]}^{\Omega^{+}, \Omega^{-}}, \quad \omega_{[a+m, b-m]^{c}}^{\Omega^{+}, \Omega^{-}}$, and $\omega^{\prime \prime}$ are non-negative functionals on $\mathcal{A}_{[a+m, b-m]}, \mathcal{A}_{[a+m, b-m]^{c}}$, and $\mathcal{A}$, respectively, defined as follows. For convenience put $\hat{\Lambda}=[a+m, b-m]$.

$$
\eta_{\hat{\Lambda}}^{\Omega^{+}, \Omega^{-}}(\cdots):=\omega_{\uparrow, \hat{\Lambda}}\left(\left(V_{\hat{\Lambda}}^{\Omega^{+}, \Omega^{-}}\right)^{*}(\cdots) V_{\hat{\Lambda}}^{\Omega^{+}, \Omega^{-}}\right),
$$

where $\omega_{\uparrow, \hat{\Lambda}}$ is the state with $S_{x}^{(3)}=S$ for all $x \in \hat{\Lambda}$, and $V_{\hat{\Lambda}}^{\Omega^{+}, \Omega^{-}}$is the unitary defined by

$$
\begin{array}{r}
V_{\hat{\Lambda}}^{\Omega^{+}, \Omega^{-}}:=\exp \left[-i \phi^{-} \sum_{x=a+m}^{b-m} S_{x}^{(3)}-\frac{i}{\hat{L}}\left(\phi^{+}-\phi^{-}\right) \sum_{x=a+m}^{b-m}(x-a-m) S_{x}^{(3)}\right] \\
\cdot \exp \left[-i \theta^{-} \sum_{x=a+m}^{b-m} S_{x}^{(2)}-\frac{i}{\hat{L}}\left(\theta^{+}-\theta^{-}\right) \sum_{x=a+m}^{b-m}(x-a-m) S_{x}^{(2)}\right]
\end{array}
$$

with $\hat{L}=b-a-2 m$, and $\Omega^{ \pm}=\left(\theta^{ \pm}, \phi^{ \pm}\right)$.

$$
\begin{array}{r}
\omega_{\hat{\Lambda}^{c}}^{\Omega^{+}, \Omega^{-}}(\cdots):=\left(\frac{2 m S+1}{4 \pi}\right)^{2} \omega_{\hat{\Lambda}^{c}}\left(Q_{[a, a+m-1]}\left(\Omega^{-}\right) Q_{[b-m+1, b]}\left(\Omega^{+}\right)(\cdots)\right. \\
\left.\cdot Q_{[a, a+m-1]}\left(\Omega^{-}\right) Q_{[b-m+1, b]}\left(\Omega^{+}\right)\right), \quad
\end{array}
$$


where $Q_{\Lambda}(\Omega)$ is defined in (5.6).

$$
\begin{array}{r}
\omega^{\prime \prime}(\cdots):=\omega_{\uparrow, \hat{\Lambda}}(\cdots) \otimes \omega_{\hat{\Lambda}^{c}}\left(\left(\mathbb{1}-P_{[a, a+m-1]} P_{[b-m+1, b]}\right)(\cdots)\right. \\
\left.\cdot\left(\mathbb{1}-P_{[a, a+m-1]} P_{[b-m+1, b]}\right)\right),
\end{array}
$$

with $P_{[k, \ell]}$ defined in (5.5).

Note that in the state $\eta_{\hat{\Lambda}}^{\Omega^{+}, \Omega^{-}}$the orientation of spins gradually rotate from $\Omega^{-}$on the left to $\Omega^{+}$on the right. Due to the known asymptotics of any ground state, the state $\omega_{\hat{\Lambda}^{c}}^{\Omega^{+}, \Omega^{-}}$, up to normalization, represents $\omega$ conditioned upon the spins having orientation $\Omega^{-}$on the sites $a, \ldots, a+m-1$ and $\Omega^{+}$on the sites $b-m+1, \ldots, b$. In finite volume all this is approximate, and the term $\omega^{\prime \prime}$ is exactly the correction needed to reproduce $\omega$ on the complement of $\Lambda$.

Before making the energy estimates, we first verify this fact, i.e., check that $\omega^{\prime}(A)=\omega(A)$ for all $A \in \mathcal{A}_{\Lambda^{c}}$. Note that the projection operators $P_{[a, a+m-1]}, P_{[b-m+1, b]}, Q_{[a, a+m-1]}\left(\Omega^{-}\right)$, and $Q_{[b-m+1, b]}\left(\Omega^{+}\right)$are elements of the algebra $\mathcal{A}_{\Lambda \cap \hat{\Lambda}^{c}}$, and therefore commute with any $A \in \mathcal{A}_{\Lambda^{c}}$ by the definitions. This fact and the definitions given above suffice to check the following for all $A \in \mathcal{A}_{\Lambda^{c}}$ :

$$
\begin{aligned}
\omega^{\prime}(A)= & \int d \Omega^{+} \int d \Omega^{-} \eta_{\hat{\Lambda}}^{\Omega^{+}, \Omega^{-}}(\mathbb{1}) \omega_{\hat{\Lambda}^{c}}^{\Omega^{+}, \Omega^{-}}(A)+\omega^{\prime \prime}(A) \\
= & \left(\frac{2 m S+1}{4 \pi}\right)^{2} \int d \Omega^{+} \int d \Omega^{-} \omega\left(Q_{[a, a+m-1]}\left(\Omega^{-}\right) Q_{[b-m+1, b]}\left(\Omega^{+}\right) A\right) \\
& +\omega\left(\left(\mathbb{1}-P_{[a, a+m-1]} P_{[b-m+1, b]}\right) A\right) \\
= & \omega\left(P_{[a, a+m-1]} P_{[b-m+1, b]} A\right)+\omega\left(\left(\mathbb{1}-P_{[a, a+m-1]} P_{[b-m+1, b]}\right) A\right) \\
= & \omega(A)
\end{aligned}
$$

Next, we estimate the energy of $\omega^{\prime}$. By definition, we have

$$
\begin{aligned}
\omega^{\prime}\left(H_{\Lambda \cup \partial \Lambda}\right) & =\int d \Omega^{+} \int d \Omega^{-} \eta_{\hat{\Lambda}}^{\Omega^{+}, \Omega^{-}}\left(H_{\hat{\Lambda}}\right) \\
& +\int d \Omega^{+} \int d \Omega^{-}\left[\omega_{\hat{\Lambda}^{c}}^{\Omega^{+}, \Omega^{-}}\left(h_{a-1, a}\right)+\omega_{\hat{\Lambda}^{c}}^{\Omega^{+}, \Omega^{-}}\left(h_{b, b+1}\right)\right] \\
& +\sum_{x=b-m}^{b} \omega^{\prime \prime}\left(h_{x, x+1}\right)+\sum_{x=a-1}^{a+m-1} \omega^{\prime \prime}\left(h_{x, x+1}\right) .
\end{aligned}
$$

First, we estimate $\eta_{\hat{\Lambda}}^{\Omega^{+}, \Omega^{-}}\left(H_{\hat{\Lambda}}\right)$. Due to the rotation invariance this quantity depends only on the angle between $\Omega^{-}$and $\Omega^{+}$. Therefore we can 
consider the case $\theta^{+}=\theta, \theta^{-}=\phi^{+}=\phi^{-}=0$. The energy is then given by the usual spin wave energy:

$$
\begin{aligned}
\eta_{\hat{\Lambda}}^{\Omega^{+}, \Omega^{-}}\left(H_{\hat{\Lambda}}\right) & =\sum_{x=a+m}^{b-m-1} \omega_{\uparrow}\left(\left(V_{\hat{\Lambda}}^{\Omega^{+}, \Omega^{-}}\right)^{*} h_{x, x+1} V_{\hat{\Lambda}}^{\Omega^{+}, \Omega^{-}}\right) \\
& =S^{2} \hat{L}[1-\cos (\theta / \hat{L})] .
\end{aligned}
$$

This implies

$$
\eta_{\hat{\Lambda}}^{\Omega^{+}, \Omega^{-}}\left(H_{\hat{\Lambda}}\right) \rightarrow 0, \text { as } \hat{L}=b-a-2 m \rightarrow \infty \text {. }
$$

Therefore the first integral in the right-hand side of (5.16) vanishes in the limit $\hat{\Lambda} \uparrow \mathbb{Z}$.

Next consider the second integral in the right-hand side of (5.16). Using the definitions (5.5), (5.6), and (5.13), we have

$$
\begin{aligned}
& \int d \Omega^{+} \int d \Omega^{-} \omega_{\hat{\Lambda}^{+}}^{\Omega^{+}, \Omega^{-}}\left(h_{b, b+1}\right) \\
& \quad=\frac{2 m S+1}{4 \pi} \int d \Omega^{+} \omega\left(Q_{[b-m+1, b]}\left(\Omega^{+}\right) h_{b, b+1} Q_{[b-m+1, b]}\left(\Omega^{+}\right) P_{[a, a+m-1]}\right) \\
& \quad \leq \omega\left(\frac{2 m S+1}{4 \pi} \int d \Omega^{+} Q_{[b-m+1, b]}\left(\Omega^{+}\right) h_{b, b+1} Q_{[b-m+1, b]}\left(\Omega^{+}\right)\right)
\end{aligned}
$$

The operator

$$
\frac{2 m S+1}{4 \pi} \int d \Omega^{+} Q_{[b-m+1, b]}\left(\Omega^{+}\right) h_{b, b+1} Q_{[b-m+1, b]}\left(\Omega^{+}\right)
$$

commutes with $\mathrm{SU}(2)$ rotations. Therefore, as any weak limit $\omega \circ \tau_{b}, b \rightarrow \infty$, is supported by the highest spin irreducible representation of $\mathrm{SU}(2)$, the limit $b \rightarrow \infty$ of (5.19) is given by

$$
\begin{aligned}
& \frac{2 m S+1}{4 \pi} \int d \Omega \omega^{(+\infty)}\left(Q_{[1, m]}(\Omega) h_{m, m+1} Q_{[1, m]}(\Omega)\right) \\
& =\frac{2 m S+1}{4 \pi} \int d \Omega \omega_{\uparrow}\left(Q_{[1, m]}(\Omega) h_{m, m+1} Q_{[1, m]}(\Omega)\right)
\end{aligned}
$$

Here again $\omega_{\uparrow}$ is the state determined by $\omega_{\uparrow}\left(S_{x}^{(3)}\right)=S$, for all $x \in \mathbb{Z}$. The 
right-hand side of (5.21) can easily be calculated:

$$
\begin{aligned}
& \frac{2 m S+1}{4 \pi} \int d \Omega \omega_{\uparrow}\left(Q_{[1, m]}(\Omega) h_{m, m+1} Q_{[1, m]}(\Omega)\right) \\
& \quad=\frac{2 m S+1}{4 \pi} \int d \Omega S^{2}\left(\cos \frac{\theta}{2}\right)^{4 m S}(1-\cos \theta) \\
& \quad=\frac{S^{2}(2 m S+1)}{2} \int_{0}^{\pi} d \theta \sin \theta\left(\frac{1+\cos \theta}{2}\right)^{2 m S}(1-\cos \theta) \\
& =\frac{S^{2}}{m S+1}
\end{aligned}
$$

Clearly this quantity vanishes as $m \rightarrow \infty$. The contribution in the second term of (5.16) coming form the left asymptotics can be estimated in the same way. Therefore, we have shown

$$
\lim _{m \rightarrow \infty} \lim _{b \rightarrow \infty} \lim _{a \rightarrow-\infty} \int d \Omega^{+} \int d \Omega^{-}\left[\omega_{\hat{\Lambda}^{c}}^{\Omega^{+}, \Omega^{-}}\left(h_{a-1, a}\right)+\omega_{\hat{\Lambda}^{c}}^{\Omega^{+}, \Omega^{-}}\left(h_{b, b+1}\right)\right]=0 .
$$

Finally, we consider the two summations in the right-hand side of (5.16). Note that

$$
\begin{aligned}
& \mathbb{1}-P_{[a, a+m-1]} P_{[b-m+1, b]}=P_{[a, a+m-1]}\left(1-P_{[b-m+1, b]}\right) \\
& \quad+\left(1-P_{[a, a+m-1]}\right) P_{[b-m+1, b]}+\left(1-P_{[a, a+m-1]}\right)\left(1-P_{[b-m+1, b]}\right) .
\end{aligned}
$$

Combining this with (5.8) and (5.14), we can conclude

$$
\lim _{b \rightarrow \infty} \lim _{a \rightarrow-\infty} \sum_{x=b-m}^{b} \omega^{\prime \prime}\left(h_{x, x+1}\right)=0, \text { and } \lim _{b \rightarrow \infty} \lim _{a \rightarrow-\infty} \sum_{x=a-1}^{a+m-1} \omega^{\prime \prime}\left(h_{x, x+1}\right)=0
$$

for a fixed $m$.

Combining (5.16), (5.18), (5.23), and (5.25), we obtain

$$
\lim _{m \rightarrow \infty} \lim _{b \rightarrow \infty} \lim _{a \rightarrow-\infty} \omega^{\prime}\left(H_{\Lambda \cup \partial \Lambda}\right)=0 .
$$

This implies (5.9).

\section{Acknowledgments}

It is a pleasure to thank the following people for discussions and correspondence: Mieke De Cock, Mark Fannes, Taku Matsui, Seiji Miyashita, Jan-Philip Solovej, Hal Tasaki, and Reinhard Werner. B.N. was partially supported by the National Science Foundation under grant \# DMS-9706599. 


\section{References}

[1] F.C. Alcaraz, S.R. Salinas, and W.F. Wreszinski, Phys. Rev. Lett. 75 (1995) 930.

[2] C.-T. Gottstein, and R.F. Werner, "Ground states of the infinite $q$ deformed Heisenberg ferromagnet", cond-mat/9501123.

[3] R. Schilling, Phys. Rev. B15 (1977) 2700.

[4] P. Prelovšek, and I. Sega, Phys. Lett. A81 (1981) 407.

[5] H.J. Mikeska, S. Miyashita, and G.H. Ristow, J. Phys. Cond. Matter 3 (1991) 2985.

[6] J.P. de Lima, and L.L. Goncalves, Mod. Phys. Lett. B8 (1994) 871.

[7] M. Henkel, A.B. Harris, and M. Cieplak, Phys. Rev. B52 (1995) 4371.

[8] M. Fannes and R.F. Werner, Helv. Phys. Acta 68 (1995) 635.

[9] T. Matsui, Lett. Math. Phys. 37 (1996) 397.

[10] I. Affleck, T. Kennedy, E.H. Lieb, H. Tasaki, Comm. Math. Phys. 115 (1988) 477.

[11] T. Matsui, Comm. Math. Phys. 189 (1997) 127.

[12] O. Bratteli, and D.W. Robinson, "Operator algebras and quantum statistical mechanics", 2 volumes, Springer-Verlag, 2nd edition, 1996.

[13] D. Babbitt, L. Thomas, Comm. Math. Phys. 54 (1977) 255.

[14] O, Bratteli, A. Kishimoto, and D. Robinson, Comm. Math. Phys. 64 (1978) 41.

[15] T. Matsui, "Positive energy representations for quantum spin models in 1+1 dimensions", preprint, Kyushu University.

[16] V. Pasquier and H. Saleur, Nucl. Phys. B330 (1990) 523.

[17] T. Koma and B. Nachtergaele, Lett. Math. Phys. 40 (1997) 1.

[18] J.R. Klauder and B.-S. Skagerstam, "Coherent states - applications in physics and mathematical physics", World Scientific 1985. 